\title{
Silicon-on-insulator polarization controller with relaxed fabrication tolerances
}

\author{
J.D. Sarmiento-Merenguel ${ }^{1}$, C. Alonso-Ramos ${ }^{1}$, R. Halir ${ }^{1}$, L. Vivien ${ }^{2}$, \\ P. Cheben ${ }^{3}$, A. Ortega-Moñux ${ }^{1}$, I. Molina-Fernández ${ }^{1}$, \\ X. Leroux ${ }^{2}$, D. Marris-Morini ${ }^{2}$, D.-X. Xu ${ }^{3}$, J. H. Schmid ${ }^{3}$, and S. Janz ${ }^{3}$ \\ 1-Universidad de Málaga, ETSI Telecomunicación, \\ Campus de Teatinos s/n, 29071 Málaga, España Email: jdsm@ic.uma.es \\ 2-Institut dElectronique Fondamentale, Univ. Paris-Sud CNRS-UMR8622, \\ Bat. 220 F-91405 ORSAY, France \\ 3-National Research Council of Canada Ottawa, K1A 0R6, Canada
}

\begin{abstract}
Polarization control is essential in applications ranging from optical communications to interferometric sensors. The implementation of integrated polarization controllers is challenging as they require polarization rotating waveguides with stringent fabrication tolerances. Here, we present a fully integrated polarization controller scheme that significantly relaxes the requirements on the rotating waveguides, alleviating fabrication tolerances. We analytically establish a technology-independent, easily measurable tolerance condition for the rotating waveguides. Polarization control in the presence of waveguide width errors of $\sim \mathbf{2 5} \%$ is shown through full vectorial simulation.
\end{abstract}

\section{Introduction}

Devices implemented on high index contrast technologies such as indium phosphide (InP) or silicon-on-insulator (SOI) are strongly polarization sensitive, so that on-chip polarization management is often required. Both integrated polarization splitters and rotators have been recently studied [1, 2]. Polarization control is of fundamental importance for polarization diversity circuits. Furthermore, enabling arbitrary transformation of the input state of polarization (SOP), is required for optical tomography applications [3]. Polarization controllers have been implemented in fiber optics [4], lithium niobate [5, 6], indium phosphide [7] and polymer waveguides [8]. However, these realizations require very accurate polarization rotators, with a polarization angle of $45^{\circ}$ and a polarization phase shift of $90^{\circ}$ or $180^{\circ}$. This results in stringent fabrication tolerances that make the implementation of these devices challenging. Here we present a polarization controller that uses tunable polarization phase shifters to substantially relax the requirements on the polarization rotation waveguides. We show by simulation that width variations up to $25 \%$ can be accommodated using this approach. 


\section{Tolerant integrated polarization controller}

Our scheme, shown in Fig. 1, is composed of three rotator waveguides sections (RWSs) and three tunable polarization phase shifters (PPSs). The RWSs are characterized by their polarization axis angle $(\theta)$, which depends on the transversal waveguide geometry, and the phase shifts $(\varphi)$ between the two orthogonal polarization states axes, which depends on the transversal waveguide geometry and the rotator length. The PPSs are defined by the phase shift $\rho$ between their TE and TM modes in the respective phase shifter sections.

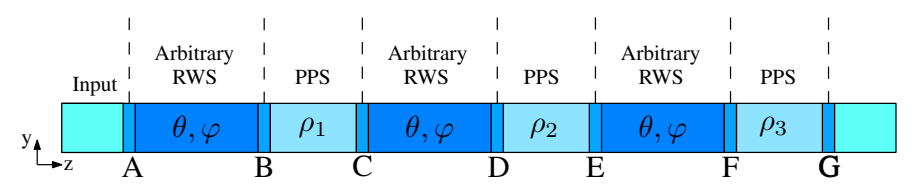

Figure 1: Schematic of the proposed polarization controller scheme comprising three arbitrary rotator waveguide sections (RWS) and three polarization phase shifters (PPS).

Using this scheme, a horizontal (TE) or vertical (TM) input SOP can be converted into an arbitrary output SOP without requiring specific rotating waveguides. To illustrate this, we consider RWSs with $\theta=27^{\circ}$ and $\varphi=103^{\circ}$ which differ significantly from the values $\theta=45^{\circ}$ and $\varphi=\left\{180^{\circ}, 90^{\circ}\right\}$ required in the conventional approaches. As a first example we convert from horizontal (TE) polarization to circular polarization by setting $\rho_{1}=281^{\circ}, \rho_{2}=64^{\circ}$ and $\rho_{3}=163^{\circ}$. Figure $2(\mathrm{a})$ shows the resulting state of polarization trace on the Poincaré sphere, where the SOPs marked A to G correspond to the interfaces in Fig. 1. It is observed that the first two PPSs are adjusted so that the fixed jumps afforded by the RWS yield an intermediate SOP from which the desired output SOP can be reached by simply adjusting the last PPS. As a second example, and in order to show that small changes in the SOP are also feasible, we consider the extreme case of horizontal to horizontal polarization conversion in Fig. 2(b). In this case PPS shifts have been adjusted to $\rho_{1}=\rho_{2}=189^{\circ}$. Note that, as the SOP at interface $\mathrm{F}$ is already horizontal, the last PPS has no effect.

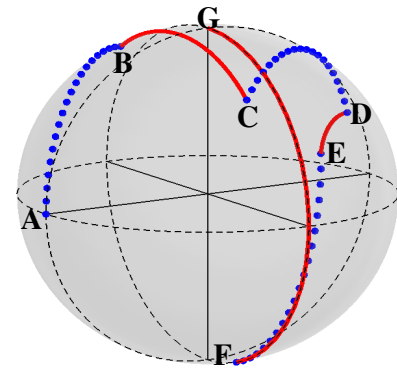

(a)

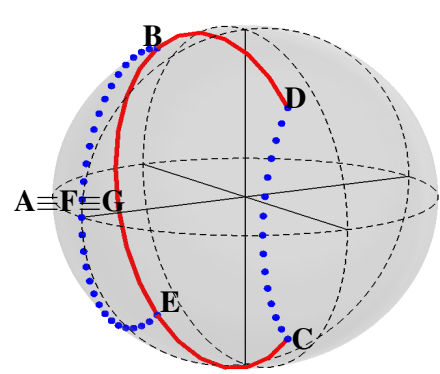

(b)

Figure 2: Poincaré sphere plot of (a) horizontal (TE) to left handed circular polarization conversion and (b) horizontal to horizontal polarization conversion (b). Points A to F corresponds to the SOPs at the interfaces A to F shown in Fig. 1. 

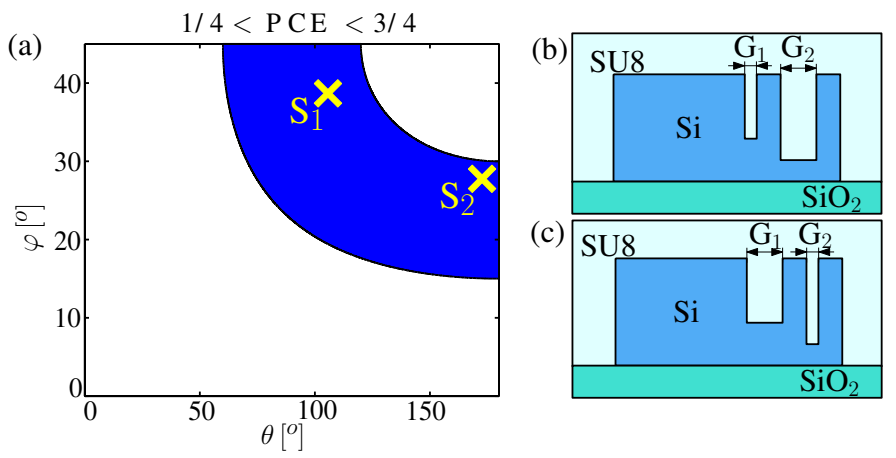

Figure 3: (a) Graphical representation of the tolerance condition (1) for full polarization control. The simulated examples are marked with $\mathrm{S}_{1}$ and $\mathrm{S}_{2}$. (b) and (c): cross section of the RWS used in simulated cases $S_{1}\left(G_{1}\right.$ narrower than nominal) and $\mathrm{S}_{2}\left(\mathrm{G}_{2}\right.$ narrower than nominal).

This example shows that full polarization control can be achieved with nonperfect RWS, i.e. $\theta \neq 45^{\circ}, \varphi \neq\left\{90^{\circ}, 180^{\circ}\right\}$. However, not all combinations of $\theta$ and $\varphi$ yield full polarization control. If the rotation effects are too "weak", it will be impossible to reach SOPs that are very different from the input SOP. A condition that ensures that the SOP opposite to the input SOP can always be reached was proposed in [9]. However, in a polarization controller, we also need to reach SOPs that are close to the input SOP, so that rotation effects should not be too "strong". By examining the Jones matrix of our polarization controller scheme (see Fig. 1), we have derived the following condition that ensures that all polarization states can be covered:

$$
1 / 4<\underbrace{\sin ^{2}(2 \theta) \sin ^{2}(\varphi / 2)}_{\mathrm{PCE}}<3 / 4 .
$$

This condition determines the set of RWS for which any output SOP can be synthesized by adjusting the three PPS. Note that Eq. (1) is a technologyindependent condition on the polarization conversion efficiency (PCE) of the individual RWS, which can be easily determined experimentally. Fig. 3(a) represents the region of $\theta$ and $\varphi$ in which the Eq. (1) is fulfilled.

To validate our approach a design example in SOI is examined through full vectorial simulations. PPSs are implemented with thermo-optic phase shifters, i.e. by heating sections of conventional Si-wire waveguides. The RWS are implemented using the double-trench approach proposed in [10] - see Fig. 3(b). The waveguides are $260 \mathrm{~nm}$ thick and $415 \mathrm{~nm}$ wide and the nominal gap widths in the rotator waveguide are $G_{1}=60 \mathrm{~nm}$ and $G_{2}=85 \mathrm{~nm}$. The three RWS are assumed to be affected by the same errors, and thus have the same dimensions. Two tolerance scenarios are considered, both falling inside the feasible working region shown in Fig. 3(a). In case $\mathrm{S}_{1}$ [Fig. 3(b)], $G_{1}$ is $20 \mathrm{~nm}$ narrower than nominal, leading to $\theta=38^{\circ}$ and $\varphi=103^{\circ}$. In case $\mathrm{S}_{2}$ [Fig. 3(c)], $G_{2}$ is $25 \mathrm{~nm}$ narrower than nominal, yielding $\theta=27.4^{\circ}$ and $\varphi=180^{\circ}$. The temperature increments of the three PPPs necessary to achieve TE, TM and left-handed circular (LHC) output SOP are obtained using full vectorial simulations in Fimmwave, and are presented in Table 1. It is observed that even in 
Table 1: Temperature changes in the PPSs required to reach different output SOPs.

\begin{tabular}{|c|c|c|c|c|}
\hline Tolerance scenario & $\Delta T_{1}$ & $\Delta T_{2}$ & $\Delta T_{3}$ & Output SOP \\
\hline $\mathrm{S}_{1}$ & 28.7 & 28.7 & - & $\mathrm{TE}$ \\
\hline $\mathrm{S}_{1}$ & 0.2 & 12.7 & 36.8 & $\mathrm{LHC}$ \\
\hline $\mathrm{S}_{1}$ & 9.7 & 26.5 & - & $\mathrm{TM}$ \\
\hline $\mathrm{S}_{2}$ & 27.2 & 27.2 & - & $\mathrm{TE}$ \\
\hline $\mathrm{S}_{2}$ & 3.5 & 5.4 & 26.8 & $\mathrm{LHC}$ \\
\hline $\mathrm{S}_{2}$ & 2.2 & 16.7 & - & $\mathrm{TM}$ \\
\hline
\end{tabular}

the presence of significant width variations all polarization states can be reached with temperature changes of $<30^{\circ} \mathrm{C}$.

\section{Conclusion}

A general polarization controller scheme with relaxed fabrication tolerances has been proposed. This polarization controller allows synthesize any state of polarization for a wide range of rotator waveguide parameters by using compensatory tunable polarization phase shifters. A technology-independent, easily measurable tolerance condition has been provided, that ensures that a specific rotator waveguide (including the fabrication errors) is suitable for full polarization control using the proposed scheme.

\section{Acknowledgments}

This work was supported by the project TEC2009-10152, and the Universidad de Málaga - Campus de Excelencia Internacional Andalucía Tech.

\section{References}

[1] D. Pérez-Galacho, R. Zhang, A. Ortega-Moñux, R. Halir, C. Alonso-Ramos, P. Runge, K. Janiak, G. Zhou, H.-G. Bach, A. G. Steffan et al., "Integrated polarization beam splitter for 100/400 GE polarization multiplexed coherent optical communications," Journal of Lightwave Technology, vol. 32, no. 3, pp. 361-368, 2013.

[2] D. Vermeulen, S. Selvaraja, P. Verheyen, P. Absil, W. Bogaerts, D. Van Thourhout, and G. Roelkens, "Silicon-on-insulator polarization rotator based on a symmetry breaking silicon overlay," Photonics Technology Letters, IEEE, vol. 24, no. 6, pp. 482-484, March 2012.

[3] M. Yamanari, Y. Lim, S. Makita, and Y. Yasuno, "Visualization of phase retardation of deep posterior eye by polarization-sensitive swept-source optical coherence tomography with $1 \mu \mathrm{m}$ probe," Opt. Express, vol. 17, pp. $12385-12396,2009$. 
[4] E. Lyons and H. Lee, "An electrically tunable all-fiber polarization controller based on deposited thin-film microheaters," Photonics Technology Letters, IEEE, vol. 14, no. 9, pp. 1318-1320, 2002.

[5] N. Walker and G. Walker, "Polarization control for coherent communications," Lightwave Technology, Journal of, vol. 8, no. 3, pp. 438-458, Mar 1990.

[6] F. Heismann, "Analysis of a reset-free polarization controller for fast automatic polarization stabilization in fiber-optic transmission systems," Lightwave Technology, Journal of, vol. 12, no. 4, pp. 690-699, Apr 1994.

[7] J. van der Tol, L. Augustin, U. Khalique, and M. Smit, "Polarization control and its application to waveguide devices," in Proc. 13th Micro optic Conf.(MOC'07), 2007, pp. 28-31.

[8] J.-W. Kim, S.-H. Park, W.-S. Chu, and M.-C. Oh, "Integrated-optic polarization controllers incorporating polymer waveguide birefringence modulators," Optics Express, vol. 20, no. 11, pp. 12 443-12 448, 2012.

[9] C. Alonso-Ramos, R. Halir, A. Ortega-Moñux, P. Cheben, L. Vivien, Í. Molina-Fernández, D. Marris-Morini, S. Janz, D.-X. Xu, and J. Schmid, "Highly tolerant tunable waveguide polarization rotator scheme," Optics Letters, vol. 37, no. 17, pp. 3534-3536, 2012.

[10] A. V. Velasco, M. L. Calvo, P. Cheben, A. Ortega-Moñux, J. H. Schmid, C. A. Ramos, Í. M. Fernandez, J. Lapointe, M. Vachon, S. Janz et al., "Ultracompact polarization converter with a dual subwavelength trench built in a silicon-on-insulator waveguide," Optics Letters, vol. 37, no. 3, pp. 365-367, 2012. 